\title{
Surface tension problems with distributed torque
}

\author{
M. Reivinen \& E.-M. Salonen \\ School of Engineering, Aalto University, Finland
}

\begin{abstract}
A formulation based on the principle of virtual work for the determination of equilibrium crystal shapes is presented. The surface tension at the interface between material phases is non-constant depending on the interface orientation. The non-constancy of the surface tension means that for interface equilibrium certain distributed torque must act on the interface. When the distributed torque has jumps in its value, edges can emerge on the interface surface. The corresponding jump conditions at the edges are derived. The presentation is mainly limited to two dimensions but the formulation can be extended rather straightforwardly also to three dimensions. A discrete formulation employing a line segment model is applied to a demonstration example having analytical solution. Further, the demonstration example with edges shows that the jump conditions are satisfied.
\end{abstract}

Keywords: crystal shape, surface tension, contact, virtual work.

\section{Introduction}

Classical methods to determine equilibrium shapes of crystals are the Wulff construction and the Landau-Andreev formulation (Nozieres [1]). The Wulff construction applies for closed shapes and does not take into account the effect of gravity. The Landau-Andreev formulation gives the interface with Cartesian coordinates in the form $z(x, y)$ and does not work with an arbitrary shaped substrate. Further, difficulties appear if the solution is double valued in $z$.

The present article describes an approach on equilibrium crystal shape determination based on the principle of virtual work. The obtained results are in accordance with reference [1] but the restrictions mentioned above do not emerge. The presentation is mainly limited to two dimensions but the 
formulation can be extended rather straightforwardly also to three dimensions. The problem statement is given in Chapter 2, the theory in Chapter 3 and the corresponding discrete approach in Chapter 4. The jump conditions are derived in Chapter 5 in the three-dimensional case and the two-dimensional case is obtained as a special case. In Chapter 6 a numerical result is presented.

\section{Problem statement}

Let us consider Figure 1. The basic setting consists of a crystal (C), surrounded by liquid (L) and resting in equilibrium on a solid surface wall (W). The task is to determine the position of the interface surface $S$ between the crystal and the liquid. The shape of the wall surface $\hat{S}$ is considered as given.

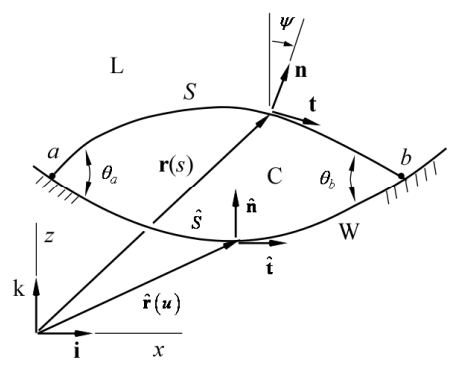

Figure 1: $\quad$ Some notations.

The study here is restricted to two dimensions and Figure 1 thus represents a cross-sectional cylindrical case. The interfacial energy density $\gamma$ depends on the orientation of the interface surface or here in this two-dimensional case on the direction angle $\psi: \gamma=\gamma(\psi)$. The interfacial energy density between the wall and the crystal phase $\gamma_{\mathrm{CW}}$ and the interfacial energy density between the wall and the liquid phase $\gamma_{\mathrm{LW}}$ depend in principle on a given way on the position on $\hat{S}$. The interface touches the wall at points $a$ and $b$, with contact angles $\theta_{a}$ and $\theta_{b}$ which are all initially unknowns of the problem. The cross-sectional area $A_{C}$ of the crystal is assumed to be given in the problem statement. Correspondingly, the pressure $p_{\mathrm{C}}$ in the crystal is an unknown constant. The known pressure in the liquid is taken according to the hydrostatic pressure distribution $p_{\mathrm{L}}=p_{0}-\rho_{\mathrm{L}} g \mathrm{z}$, where $\rho_{\mathrm{L}}$ is the constant density of the liquid, $g$ the acceleration of gravity, $z$ the upwards measured coordinate and $p_{0}$ a given pressure associated with the level $z=0$. When the hydrostatic pressure distribution is applied on $S, z$ must naturally be measured on the surface. The position vector $\mathbf{r}$ on $S$ is expressed in the form $\mathbf{r}=\mathbf{r}(s)$ where the $s$ is arc length. The position vector $\hat{\mathbf{r}}$ on $\hat{S}$ is expressed (sometimes in a piecewise 
manner) in the form $\hat{\mathbf{r}}=\hat{\mathbf{r}}(u)$ where $u$ is a suitable curve parameter. The unit normal vectors $\mathbf{n}$ and $\hat{\mathbf{n}}$ on $S$ and $\hat{S}$ are directed into the liquid and into the crystal and the unit tangent vectors $\mathbf{t}$ and $\hat{\mathbf{t}}$ into the increasing directions of $s$ and $u$, respectively.

\section{Virtual work}

The principle of virtual work is a well-known formulation with wide application areas in mechanics, e.g. Lanczos [2]. We write the principle down for the interface problem under study and then show finally that the relevant equations follow from it. The virtual work equation is

$$
\delta^{\prime} W \equiv \delta^{\prime} W^{\text {int }}+\delta^{\prime} W^{\text {ext }}+\delta^{\prime} W^{\text {bound }}=0,
$$

where $\delta^{\prime} W^{\text {int }}$ is the virtual work of the internal forces, $\delta^{\prime} W^{\text {ext }}$ is the virtual work of the external forces and $\delta^{\prime} W^{\text {bound }}$ is the virtual work of from the boundary terms. The principle of virtual work states that (1) is valid for any virtual movement of the interface. The principle of virtual work is not a variational principle in the sense that a stationarity condition of a functional is not involved. The prime on $\delta$ is used to emphasize thus that no variations of some quantities $W$ are involved. Usually in the principle of virtual work the variation of the position vector of material particles are involved and the variation is called virtual displacement. Here the change in the position of the interface will be called virtual movement to indicate that the movement is not necessarily associated with a material particle. The term $\delta^{\prime} W^{\text {bound }}$ could be included in the virtual work of the external forces but because of its rather special form it has been included as a separate term. Finally, although the interface problem is according to Nozieres [1] a "chemical” problem, we mostly borrow terminology from mechanics.

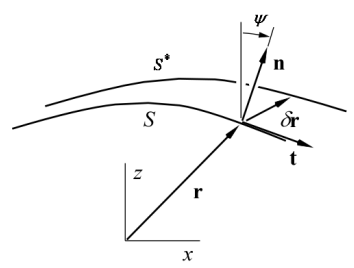

Figure 2: $\quad$ Position vector $\mathbf{r}$ and virtual movement $\delta \mathbf{r}$.

Let us consider Figure 2. Let the position vector to a generic point on the interface curve $S$ be denoted $\mathbf{r}(s)$ and let the corresponding point on a varied comparison curve $S^{*}$ be given by $\mathbf{r}(s)+\delta \mathbf{r}(s)$, where $\delta \mathbf{r}$ is called here virtual movement. The expression for the virtual work of internal forces is 


$$
\delta^{\prime} W^{\text {int }}=-\int_{s} \gamma \mathbf{t} \cdot \frac{\mathrm{d} \delta \mathbf{r}}{\mathrm{d} s} \mathrm{~d} s
$$

The integral is over the interface curve from $a$ to $b$.

The external "forces" acting on the interface consist of the pressure difference $\left(p_{\mathrm{C}}-p_{\mathrm{L}}\right) \mathbf{n}$ and of a torque $\mathbf{m}=m \mathbf{j}$, where $\mathbf{j}$ is the unit vector in the $y$-axis direction. The physical unit of $m$ is the same as of $\gamma: \mathrm{N} / \mathrm{m}$. The virtual work of external forces is given by

$$
\bar{\delta} W^{\mathrm{ext}}=\int_{s}\left(p_{\mathrm{C}}-p_{\mathrm{L}}\right) \mathbf{n} \cdot \delta \mathbf{r} \mathrm{d} s-\int_{s} m \mathbf{n} \cdot \frac{\mathrm{d} \delta \mathbf{r}}{\mathrm{d} s} \mathrm{~d} s .
$$

We consider next the constraint of the given cross-section area of the crystal. This can be expressed as

$$
A \equiv \frac{1}{2} \int_{S} \mathbf{r} \cdot \mathbf{n} \mathrm{d} s-\frac{1}{2} \int_{u} \hat{\mathbf{r}} \cdot \hat{\mathbf{n}} h \mathrm{~d} u=A_{C} .
$$

The second integral is over the domain of $u$ corresponding to points $a$ and $b$. The scale factor $h=\sqrt{(\mathrm{d} x / \mathrm{d} u)^{2}+(\mathrm{d} z / \mathrm{d} u)^{2}}$. Variation of (4) gives after some manipulation the equation $\int_{S} \mathbf{n} \bullet \delta \mathbf{r d} s=0$. If this varied constraint is included in the virtual work equation by using the Lagrange multiplier method, the left-hand side of (1) obtains the contribution

$$
\lambda \int_{s} \mathbf{n} \cdot \delta \mathbf{r d} s,
$$

where $\lambda$ is the Lagrange multiplier. However, then (3) must be written originally without the term associated with $p_{\mathrm{C}}$. Comparison of (3) and (5) shows that we have the interpretation $\lambda=p_{\mathrm{C}}$ and we can continue using just the symbol $p_{\mathrm{C}}$ and the expression (3).

The rather non-transparent virtual work expression from the boundary points is found to be $\left(s_{a}=0\right)$

$$
\begin{aligned}
\bar{\delta} W^{\text {bound }}= & -\left.\gamma \frac{\hat{\mathbf{n}} \cdot \delta \mathbf{r}}{\sin \theta}\right|_{s=s_{a}}+\left.\left(\gamma_{\mathrm{LW}}-\gamma_{\mathrm{CW}}\right) \frac{\mathbf{n} \cdot \delta \mathbf{r}}{\sin \theta}\right|_{s=s_{a}} \\
& -\left.\gamma \frac{\hat{\mathbf{n}} \cdot \delta \mathbf{r}}{\sin \theta}\right|_{s=s_{b}}+\left.\left(\gamma_{\mathrm{LW}}-\gamma_{\mathrm{CW}}\right) \frac{\mathbf{n} \cdot \delta \mathbf{r}}{\sin \theta}\right|_{s=s_{b}},
\end{aligned}
$$

where from the geometry at $a$ and $\left.b \quad \hat{\mathbf{n}}\right|_{s=s_{a}}=\left.(\sin \theta \mathbf{t}+\cos \theta \mathbf{n})\right|_{s=s_{a}}$, $\left.\hat{\mathbf{n}}\right|_{s=s_{b}}=\left.(-\sin \theta \mathbf{t}+\cos \theta \mathbf{n})\right|_{s=s_{b}}$. In (6), it is not necessary that the virtual movement $\delta \mathbf{r}$ is such that the end points remain on the wall surface. However, if $\delta \mathbf{r}$ selected so, the first terms on the right-hand sides of (6) are seen to vanish. 
When $a$ or $b$ is not fixed in position in advance, we call the boundary as free. If the physics demand point $a$ or $b$ fixed in position, the corresponding is $\delta \mathbf{r}$ set to zero and no contribution to the virtual work equation appears. form

Collecting all the terms given above, the virtual work equation (1) obtains the

$$
\begin{aligned}
-\int_{s} \gamma \mathbf{t} \cdot \frac{\mathrm{d} \delta \mathbf{r}}{\mathrm{d} s} \mathrm{~d} s+\int_{s}\left(p_{\mathrm{C}}-p_{\mathrm{L}}\right) \mathbf{n} \cdot \delta \mathbf{r} \mathrm{d} s-\int_{s} m \mathbf{n} \cdot \frac{\mathrm{d} \delta \mathbf{r}}{\mathrm{d} s} \mathrm{~d} s \\
-\left.\gamma \frac{\hat{\mathbf{n}} \cdot \delta \mathbf{r}}{\sin \theta}\right|_{s=s_{a}}+\left.\left(\gamma_{\mathrm{LW}}-\gamma_{\mathrm{CW}}\right) \frac{\mathbf{n} \cdot \delta \mathbf{r}}{\sin \theta}\right|_{s=s_{a}} \\
-\left.\gamma \frac{\hat{\mathbf{n}} \cdot \delta \mathbf{r}}{\sin \theta}\right|_{s=s_{b}}+\left.\left(\gamma_{\mathrm{LW}}-\gamma_{\mathrm{CW}}\right) \frac{\mathbf{n} \cdot \delta \mathbf{r}}{\sin \theta}\right|_{s=s_{b}}=0 .
\end{aligned}
$$

Borrowing the terminology used especially in the finite element literature, e.g. Belytschko et al. [3], (7) is a weak formulation of the present problem. Based on the arbitrariness of $\delta \mathbf{r}$, we can deduce the corresponding so-called strong forms from it. The necessary manipulations are based on integration by parts yielding finally the field equations

$$
p_{\mathrm{C}}-p_{\mathrm{L}}=\frac{\gamma}{R}-\frac{\mathrm{d} m}{\mathrm{~d} s}, \quad s_{\mathrm{a}}<s<s_{\mathrm{b}}
$$

and

$$
\frac{\mathrm{d} \gamma}{\mathrm{d} s}+\frac{m}{R}=0, \quad s_{\mathrm{a}}<s<s_{\mathrm{b}} .
$$

The derivation of equations (8) and (9) from the weak form (7) is treated in more detail in [4]. Further, the following boundary conditions are arrived at

$$
\begin{aligned}
& \gamma \cos \theta-m \sin \theta=\gamma_{\mathrm{LW}}-\gamma_{\mathrm{CW}}, \quad s=s_{a}, \\
& \gamma \cos \theta+m \sin \theta=\gamma_{\mathrm{LW}}-\gamma_{\mathrm{CW}}, \quad s=s_{b} .
\end{aligned}
$$

From (9) follows

$$
m=-\frac{\mathrm{d} \gamma}{\mathrm{d} \psi} .
$$

This important result shows that for equilibrium in the tangential direction, the torque cannot be arbitrary but should be evaluated according to (12).

Using relation (12) in equation (8) gives now

$$
p_{\mathrm{C}}-p_{\mathrm{L}}=\frac{\gamma+\mathrm{d}^{2} \gamma / \mathrm{d} \psi^{2}}{R}
$$

as the final equilibrium equation in the normal direction. The term $\gamma+\mathrm{d}^{2} \gamma / \mathrm{d} \psi^{2}$ is called the surface stiffness. 


\section{Discrete formulation}

The discrete formulation is based on a kind of finite element method. The interface is discretized by two-noded line elements or segments. Figure 3 shows the notations for a generic line segment. The position vector along the segment is given by

$$
\mathbf{r}=(1-\xi) \mathbf{r}_{1}+\xi \mathbf{r}_{2}=\left[(1-\xi) x_{1}+\xi x_{2}\right] \mathbf{i}+\left[(1-\xi) z_{1}+\xi z_{2}\right] \mathbf{k},
$$

where instead of the local arc length $s$ the dimensionless coordinate $\xi=s / L$ is used. $L$ is the length of the segment.

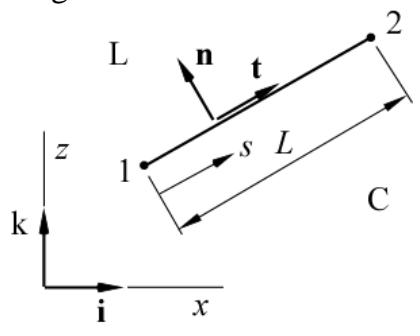

Figure 3: A generic line segment.

The generalized coordinates used are (normally) the nodal Cartesian coordinates $x_{1}, z_{1}, x_{2}, z_{2}$. From (14) by variation, the virtual movement along the segment is

$$
\delta \mathbf{r}=(1-\xi) \delta \mathbf{r}_{1}+\xi \delta \mathbf{r}_{2}=\left[(1-\xi) \delta x_{1}+\xi \delta x_{2}\right] \mathbf{i}+\left[(1-\xi) \delta z_{1}+\xi \delta z_{2}\right] \mathbf{k} .
$$

The virtual work contribution from the segment obtains finally the form

$$
\delta^{\prime} W=X_{1} \delta x_{1}+Z_{2} \delta z_{1}+X_{2} \delta x_{2}+Z_{2} \delta z_{2},
$$

where $X_{1}, Z_{1}, X_{2}, Z_{2}$ are generalized forces. The generalized forces consist similarly as the virtual work from terms corresponding to the internal and the external forces and to the free boundary. The derivation of the terms is discussed in more detail in [4].

The contribution to the cross-sectional area from the segment is simply (using expression (4))

$$
\Delta A=\frac{1}{2}\left(x_{2} z_{1}-x_{1} z_{2}\right) .
$$

The geometry of the discrete interface model is fixed by a list consisting of the nodal Cartesian coordinates, say $\mathbf{X}$, and of the global node numbers of the segments in the model. The task is to determine that $\mathbf{X}$ for which the main system equations (equilibrium equations) are satisfied. An additional unknown is the pressure $p_{\mathrm{C}}$ in the crystal. The corresponding additional system equation is the area constraint $A=A_{C}$. It is to be noted that $p_{C}$ appears in the equilibrium 
equations but not in the constraint equation. As the interface determination problem is strongly non-linear, the solution must be found iteratively.

The generalized coordinates (which might be called also generalized movements) are denoted here $q_{i}$. They are defined in principle anew for the each current system configuration. Normally, we will employ two movements $\Delta x_{k}$ and $\Delta z_{k}$ for a generic node $k$ inside the mesh. However, at a free boundary node, only one movement in the solid surface tangent direction is used.

The virtual work for the interface model (with respect to a current configuration) obtains the form

$$
\delta^{\prime} W=\sum_{i=1}^{N_{\mathrm{dof}}} Q_{i} \delta q_{i},
$$

where $Q_{i}$ is the $i$ :th generalized force corresponding to the $i$ :th movement $q_{i}$ and $N_{\text {dof }}$ the total number of movements. The generalized forces must vanish and the area constraint must be satisfied. Thus, the system equations are

$$
\begin{aligned}
& Q_{i}=0, \quad i=1,2, \cdots N_{\mathrm{dof}}, \\
& A=A_{\mathrm{C}} .
\end{aligned}
$$

More specifically, corresponding to a generic node $k$ inside the mesh where the movements $\Delta x_{k}$ and $\Delta z_{k}$ are used, the corresponding two discrete equilibrium equations in (19) are

$$
\begin{aligned}
& X_{k}=0, \\
& Z_{k}=0 .
\end{aligned}
$$

For a given $\overline{\mathbf{X}}$ and $\bar{p}_{\mathrm{C}}$ - initially guessed or updated - the system equations (19) are naturally usually not satisfied. Let the corresponding values of the lefthand sides in (19) $\bar{Q}_{i}$ and $\bar{A}$. The movements $q_{j}$ and the pressure change $\Delta p_{\mathrm{C}}$ needed towards a hopefully better solution are determined using an application of the Newton-Raphson method, which is presented in [4].

\section{Jump conditions}

When the distributed torque has jumps in its value, edges can emerge on the interface surface. For future use, the derivation of the jump conditions at the edges is performed in the three-dimensional case. The two-dimensional case is obtained as a special case.

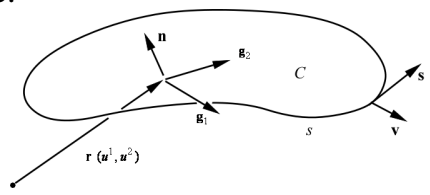

Figure 4: $\quad$ Interface surface $C$ and some notations. 
The position vector $\mathbf{r}$ to interface surface $C$ is represented as $\mathbf{r}=\mathbf{r}\left(u^{1}, u^{2}\right)$, where $u^{1}$ and $u^{2}$ are the surface parameters. Of course, this relationship is originally unknown. Here and later we will follow rather closely the notations of reference [5] and also employ some relevant formulas presented there.

Again, the surface tension $\gamma$ is not constant but depends in a prescribed way on the orientation of the interface and consequently on position. The equilibrium of the interface is then not possible without a distributed torque loading $\mathbf{~ m}$.

The three-dimensional counterpart to virtual work equation (7) can be written as

$$
\begin{aligned}
\delta^{\prime} W \equiv & -\int_{u^{1}, u^{2}} \gamma\left[\left(\mathbf{n} \times \mathbf{g}_{1}\right) \cdot \frac{\partial \delta \mathbf{r}}{\partial u^{2}}-\left(\mathbf{n} \times \mathbf{g}_{2}\right) \cdot \frac{\partial \delta \mathbf{r}}{\partial u^{1}}\right] \mathrm{d} u^{1} \mathrm{~d} u^{2} \\
& +\int_{u^{1}, u^{2}}\left(m_{1} \mathbf{n} \cdot \frac{\partial \delta \mathbf{r}}{\partial u^{2}}-m_{2} \mathbf{n} \cdot \frac{\partial \delta \mathbf{r}}{\partial u^{1}}\right) \mathrm{d} u^{1} \mathrm{~d} u^{2} \\
& +\int_{u^{1}, u^{2}} \sqrt{g}\left(p_{\mathrm{C}}-p_{\mathrm{L}}\right) \mathbf{n} \cdot \delta \mathbf{r} \mathrm{d} u^{1} \mathrm{~d} u^{2}+b t=0 .
\end{aligned}
$$

Above, the first integral is the virtual work of the internal forces, the second integral is the external virtual work from the torque (moment per unit interface surface) $\mathbf{m}=m_{1} \mathbf{g}^{1}+m_{2} \mathbf{g}^{2}$, and the third integral is the external virtual work from the pressure difference $p_{\mathrm{C}}-p_{\mathrm{L}}$. The term $b$ t refers to the virtual work from the free boundary and is not given here in detail as it does not affect the jump conditions. Further explanations concerning equation (21) are as follows. The integrals are over the appropriate domain in the $u^{1} u^{2}$ - plane. $\delta \mathbf{r}$ is the virtual movement from surface $C$ to a varied surface $C^{*}$. $\mathbf{n}$ is the unit normal vector to C. $\mathbf{g}_{1}$ and $\mathbf{g}_{2}$ are the covariant and $\mathbf{g}^{1}$ and $\mathbf{g}^{2}$ the contravariant basis vectors and $g$ is the discriminant of the covariant metric tensor. Further, $\mathbf{s}$ is the unit vector tangent to the boundary line and $\mathbf{v}=v_{1} \mathbf{g}^{1}+v_{2} \mathbf{g}^{2}$ the unit vector in the tangent plane and normal to the boundary.

Based on the arbitrariness of the virtual movement $\delta \mathbf{r}$, we can deduce similarly as shown in Chapter 3 in the two-dimensional case the strong forms from the weak formulation (21) of the present problem. For obtaining the strong forms, the derivatives of $\delta \mathbf{r}$ with respect to $u^{1}$ and $u^{2}$ must be removed by integration by parts. Some development of the formula (1.13.61) in [6] gives the appropriate integration by parts formula producing the line integral contribution

$$
\begin{aligned}
L \equiv & -\int_{s} \frac{\gamma}{\sqrt{g}}\left[v_{2}\left(\mathbf{n} \times \mathbf{g}_{1}\right)-v_{1}\left(\mathbf{n} \times \mathbf{g}_{2}\right)\right] \cdot \delta \mathbf{r} \mathrm{d} s \\
& +\int_{s} \frac{1}{\sqrt{g}}\left[\left(v_{2} m_{1}-v_{1} m_{2}\right) \mathbf{n}\right] \cdot \delta \mathbf{r} \mathrm{d} s .
\end{aligned}
$$

We will not derive here all the strong forms following from (21). For our purpose it is enough to consider just (22) further. First, 


$$
\mathbf{n} \times \mathbf{g}_{1}=\sqrt{g} \mathbf{g}^{2}, \quad \mathbf{n} \times \mathbf{g}_{2}=-\sqrt{g} \mathbf{g}^{1},
$$

so

$$
v_{2}\left(\mathbf{n} \times \mathbf{g}_{1}\right)-v_{1}\left(\mathbf{n} \times \mathbf{g}_{2}\right)=\sqrt{g}\left(v_{2} \mathbf{g}^{2}+v_{1} \mathbf{g}^{1}\right)=\sqrt{g} \mathbf{v} .
$$

Second,

$$
\mathbf{s}=\mathbf{n} \times \mathbf{v}=\mathbf{n} \times\left(v_{1} \mathbf{g}^{1}+v_{2} \mathbf{g}^{2}\right)=v_{1} \mathbf{n} \times \mathbf{g}^{1}+v_{2} \mathbf{n} \times \mathbf{g}^{2}=\frac{1}{\sqrt{g}}\left(v_{1} \mathbf{g}_{2}-v_{2} \mathbf{g}_{1}\right) .
$$

and thus

$$
\mathbf{m} \cdot \mathbf{s}=\left(m_{1} \mathbf{g}^{1}+m_{2} \mathbf{g}^{2}\right) \cdot \frac{1}{\sqrt{g}}\left(v_{1} \mathbf{g}_{2}-v_{2} \mathbf{g}_{1}\right)=\frac{1}{\sqrt{g}}\left(-m_{1} v_{2}+m_{2} v_{1}\right) .
$$

When (24) and (26) are taken into account in (22), we arrive at the formula

$$
L=-\int_{S}[\gamma \mathbf{v}+(\mathbf{m} \cdot \mathbf{s}) \mathbf{n}] \cdot \delta \mathbf{r} \mathrm{d} s .
$$

The expression inside the brackets is physically rather transparent. Especially, the term $\mathbf{m} \cdot \mathbf{s}$ is the scalar component of the torque vector $\mathbf{m}$ along the boundary tangent direction.

The above result was obtained assuming that the surface $C$ is smooth. Let us consider the case where there is an edge along a line $c$ shown in Figure 5.

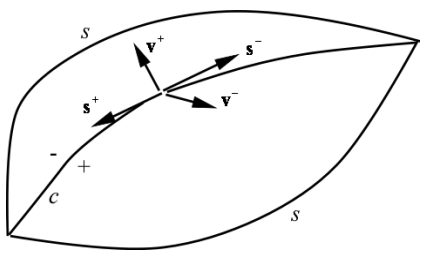

Figure 5: An edge.

Now the integration by parts manipulation must be performed in a piecewise manner. The values of the quantities on both sides of $c$ are indicated by minus and plus superscripts as shown in the figure. (Here $\mathbf{s}^{+}=-\mathbf{s}^{-}$. Vectors $\mathbf{n}^{-}$and $\mathbf{n}^{+}$are not drawn to keep the figure simple enough.) Two contributions like (27) appear along $c$ and we obtain together the term

$$
\int_{C}\left[-\gamma^{-} \mathbf{v}^{-}-\left(\mathbf{m}^{-} \cdot \mathbf{s}^{-}\right) \mathbf{n}^{-}-\gamma^{+} \mathbf{v}^{+}-\left(\mathbf{m}^{+} \cdot \mathbf{s}^{+}\right) \mathbf{n}^{+}\right] \cdot \delta \mathbf{r} \mathrm{d} s .
$$

As the virtual movement is arbitrary, the jump conditions on $c$ become

$$
-\gamma^{-} \mathbf{v}^{-}-\left(\mathbf{m}^{-} \cdot \mathbf{s}^{-}\right) \mathbf{n}^{-}-\gamma^{+} \mathbf{v}^{+}-\left(\mathbf{m}^{+} \cdot \mathbf{s}^{+}\right) \mathbf{n}^{+}=\mathbf{0}
$$


Jump conditions in two-dimensional case can be obtained from (29) as follows. Figure 6 shows a cross-section of the interface at an angular point. Now $\mathbf{t}^{-}$and $\mathbf{t}^{+}$are the tangent unit vectors. The torque is here $\mathbf{m}=m \mathbf{j}$. Let $\mathbf{s}^{-}=\mathbf{j}$ and thus $\mathbf{s}^{+}=-\mathbf{j}$. Then

$$
\mathbf{m}^{-} \cdot \mathbf{s}^{-}=m^{-} \mathbf{j} \cdot \mathbf{j}=m^{-}, \quad \mathbf{m}^{+} \cdot \mathbf{s}^{+}=m^{+} \mathbf{j} \cdot(-\mathbf{j})=-m^{+} .
$$

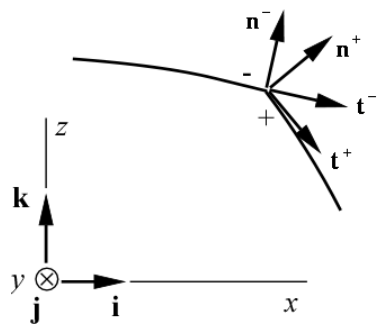

Figure 6: An angular point in two-dimensional case.

Further, comparing Figures 5 and $6, \mathbf{v}^{-}=\mathbf{t}^{-}$and $\mathbf{v}^{+}=-\mathbf{t}^{+}$. The jump conditions (29) obtain thus here finally the form

$$
-\gamma^{-} \mathbf{t}^{-}-m^{-} \mathbf{n}^{-}+\gamma^{+} \mathbf{t}^{+}+m^{+} \mathbf{n}^{+}=\mathbf{0} .
$$

\section{Numerical example}

A simple two-dimensional example case having analytical solution is presented to give an idea about the working of the discrete method. The wall is a corner consisting of the planes $z=0$ and $x=0$. The interface surface energy expression is from Junes [7]:

$$
\gamma=\left(|\sin \psi|+|\cos \psi|+\frac{1}{2}\left|\sin \left(\frac{\pi}{4}+\psi\right)\right|+\frac{1}{2}\left|\cos \left(\frac{\pi}{4}+\psi\right)\right|\right) \gamma_{0},
$$

where $\gamma_{0}$ is a reference value. Constant values $\gamma_{\mathrm{LW}}=\gamma_{\mathrm{CW}}$ were used giving the contact angles $\theta=\pi / 2$. The corresponding Wulff construction solution is shown in Figure 7 (a). The interface consists just of straight lines. Now the discontinuities in the torque appear at $\psi=0, \psi= \pm \pi / 4, \psi= \pm \pi / 2$, $\psi= \pm 3 \pi / 4, \psi= \pm \pi$.

Let us consider the upper angular point in Figure 7 (a). We have $\mathbf{n}^{-}=\mathbf{k}$, $\mathbf{t}^{-}=\mathbf{i}, \quad \mathbf{n}^{+}=\mathbf{i} / \sqrt{2}+\mathbf{k} / \sqrt{2}, \quad \mathbf{t}^{+}=\mathbf{i} / \sqrt{2}-\mathbf{k} / \sqrt{2} \quad$ and $\quad \gamma^{-}=(1+1 / \sqrt{2}) \gamma_{0}$, $m^{-}=-\gamma_{0} \quad \gamma^{+}=(1 / 2+\sqrt{2}) \gamma_{0}, m^{+}=\gamma_{0} / 2$. When these values are substituted in (31), the equation is found to be satisfied. The present problem has been solved also by the discrete approach described in [4]. The results of the 
calculations are shown in Figure 7 (b). The number of segments has been 14 . The initial guess for the interface is smooth (dashed line). It is interesting to note that the solution process so to say automatically finds the angular solution (solid line) in a rather satisfactory way. Further example cases are given in [4].
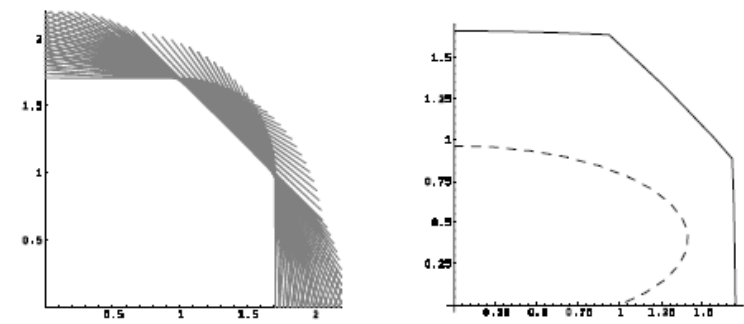

Figure 7: $\quad$ (a) Wulff construction; (b) Discrete solution.

\section{Conclusions}

The results obtained in this study and in [4] show that the presented formulation based on the principle of virtual work is correct. The initial geometries to start the calculations can be rather arbitrary and far from the final ones, and the discrete solutions have still been found in general to converge. Further, the discrete solution approach has been able to mimic the possible angular behaviour of the solution. The jump conditions in surface tension problems with distributed torque have been derived in the three-dimensional case. As a special case, the jump conditions are then obtained in the two-dimensional case. The example case in two dimensions demonstrates that the derived jump conditions are satisfied at the angular points.

\section{References}

[1] P. Nozieres, Shape and growth of crystals, in Solids far from equilibrium, Cambridge University Press, edited by C. Godreche, (1989).

[2] C. Lanczos, The Variational Principles of Mechanics, fourth ed, University of Toronto Press, (1970).

[3] T. Belytschko, W. K. Liu, B. Moran. Nonlinear Finite Elements for Continua and Structures, Wiley, 2000.

[4] Mika Reivinen, Eero-Matti Salonen, Igor Todoshchenko, Vesa P. Vaskelainen, Equilibrium crystal shapes by virtual work, J Low Temp Phys (2013) 170:75-90.

[5] E. Kreyszig. Differential Geometry, Dover, 1991.

[6] A. E. Green, W. Zerna, Theoretical Elasticity, second edition, Dover, 1968.

[7] H. Junes, Liquid-Solid Interference of 3He Imaged Using a Fabry-Pérot Interferometer, Master's Thesis, Helsinki University of Technology, 2004. 